\title{
Produção de Mudas de Ipê-amarelo (Tabebuia serratifolia) em Resposta a Fertilização Nitrogenada
}

\author{
Lívia Mara Lima Goulart ${ }^{1}$, Haroldo Nogueira de Paiva ${ }^{2}$, Hélio Garcia Leite ${ }^{2}$, \\ Aloisio Xavier ${ }^{2}$, Marciel Lelis Duarte ${ }^{2}$ \\ ${ }^{1}$ Departamento de Solos e Recursos Ambientais, Universidade Estadual Paulista - UNESP, Botucatu/SP, Brasil \\ ${ }^{2}$ Departamento de Engenharia Florestal, Universidade Federal de Viçosa - UFV, Viçosa/MG, Brasil
}

\begin{abstract}
RESUMO
Este trabalho objetivou avaliar o efeito de fontes de nitrogênio e respectivas doses sobre o crescimento e a qualidade de mudas de ipê-amarelo (Tabebuia serratifolia). As mudas foram produzidas em Latossolo Vermelho-Amarelo e as fontes de nitrogênio utilizadas foram nitrato de amônio, sulfato de amônio e nitrato de cálcio, aplicadas em cinco doses $(0 \mathrm{mg}, 75 \mathrm{mg}, 150 \mathrm{mg}, 225 \mathrm{mg}$ e $300 \mathrm{mg} \mathrm{dm}^{-3}$ ) e parceladamente, aos 25, 50, 75 e 100 dias após a repicagem. Adotou-se esquema fatorial $3 \times 5$ no delineamento experimental, casualizado em blocos, com quatro repetições. Aos 125 dias após a repicagem, a adição do nitrogênio proporcionou efeito significativo na produção de mudas de ipê-amarelo, sendo que a fonte sulfato de amônio produziu as melhores médias para todas as características e índices avaliados. Recomenda-se, para a produção de mudas de ipê-amarelo, a utilização de sulfato de amônio na dose de $100 \mathrm{mg} \mathrm{dm}^{-3}$ de $\mathrm{N}$.
\end{abstract}

Palavras-chave: nutrição florestal, propagação de plantas, espécies nativas, nitrogênio.

\section{Production of Yellow Cortez (Tabebuia serratifolia) Seedlings in Response to Nitrogen Fertilization}

\begin{abstract}
This work aimed to evaluate the effect of sources of nitrogen and respective levels on growth and quality of Yellow Cortez (Tabebuia serratifolia) seedlings. Seedlings were grown on a Oxisol and nitrogen sources were ammonium nitrate, ammonium sulphate and calcium nitrate, applied in five doses $\left(0,75,150,225\right.$ and $\left.300 \mathrm{mg} \mathrm{dm}^{-3}\right)$ and increments of 25, 50, 75 and 100 days after transplanting. The experimental design was randomized in blocks in factorial scheme $(3 \times 5)$ with four replications. At 125 days after transplanting, the addition of nitrogen provided significant effect on seedling production of Yellow Cortez (Tabebuia serratifolia), and the ammonium sulfate produced the best means for all traits and indexes evaluated. We recommend the use of ammonium sulfate at a dose of $100 \mathrm{mg} \mathrm{dm}^{-3} \mathrm{~N}$ for Yellow Cortez seedling.
\end{abstract}

Keywords: forest nutrition, plant propagation, native species, nitrogen. 


\section{INTRODUÇÃO}

O ipê-amarelo (Tabebuia serratifolia Vahl Nich.), pertencente à família Bignoniaceae, é uma espécie arbórea que atinge de 5 a $20 \mathrm{~m}$ de altura (Lorenzi, 1992) e possui interesse econômico madeireiro, ornamental e medicinal. A madeira é empregada em marcenaria, construções pesadas e estruturas externas, tanto civis quanto navais (Ferreira et al., 2004). Suas flores foram declaradas símbolo do Brasil pelo então presidente Jânio Quadros, ressaltando assim o valor cultural dessa árvore no território nacional (Cruz, 1965).

A propagação do ipê-amarelo é feita por meio de sementes que, apesar de produzidas em grande quantidade, apresentam problemas de germinação e de conservação. A dificuldade na germinação das sementes dessa espécie tem causado uma diminuição de sua ocorrência natural no Brasil (Oliveira et al., 2005), assim torna-se essencial um bom manejo silvicultural das mudas no viveiro para se evitarem perdas e proporcionar alta qualidade das mudas.

A boa formação das mudas no viveiro também contribui para o sucesso do plantio no campo, sendo que o teor de nutrientes no substrato, principalmente nitrogênio, fósforo e potássio, tem grande influência na qualidade das mudas produzidas e no desenvolvimento delas em campo (Tucci et al., 2009).

Estudos sobre a fertilização nitrogenada de mudas de espécies nativas têm evidenciado respostas positivas na produção de mudas de sete-cascas, fedegoso, canafístula, angico-vermelho, sansão-do-campo, sabiá, jacarandá-da-Bahia, jacaré e espécies florestais diversas, (Cruz et al., 2006, 2010, 2012; Gonçalves et al., 2008, 2010, 2013; Marques et al., 2006a, 2009; Souza et al., 2006).

Neste trabalho objetivou-se avaliar o efeito de fontes e de doses de nitrogênio sobre o crescimento e a qualidade de mudas de ipê-amarelo (Tabebuia serratifolia).

\section{MATERIAL E MÉTODOS}

O experimento foi conduzido no município de Viçosa, estado de Minas Gerais, no Viveiro de Pesquisas do Departamento de Engenharia Florestal da Universidade Federal de Viçosa - DEF/UFV, no período de novembro de 2010 a maio de 2011. As médias diárias de temperatura, precipitação e umidade relativa do ar atingidas no período foram de $21{ }^{\circ} \mathrm{C}, 8,8 \mathrm{~mm}$ e $81,3 \%$, respectivamente.

O solo utilizado como substrato para a produção das mudas foi um Latossolo Vermelho-Amarelo, classe predominante na região da Zona da Mata de Minas Gerais. As amostras foram retiradas de cerca de $30 \mathrm{~cm}$ abaixo da superfície e caracterizadas quimicamente (Tabela 1) e quanto à granulometria ( $14 \%$ de areia grossa, $8 \%$ de areia fina, $10 \%$ de silte e $68 \%$ de argila de classe textural muito argilosa).

O solo teve sua acidez corrigida pela saturação por bases elevada a $60 \%$, por uma mistura de $\mathrm{CaCO}_{3}$ e $\mathrm{MgCO}_{3}$, na relação estequiométrica de 4:1, conforme Gonçalves et al. (2000) ficando incubado por um período de 30 dias, com umidade mantida próxima à capacidade de campo.

Após o período de incubação com o calcário, $1,5 \mathrm{dm}^{3}$ de solo foi colocado em cada vaso, recebendo fertilização básica com macronutrientes via solução, nas doses de $300 \mathrm{mg} \mathrm{dm}^{-3} \mathrm{de} \mathrm{P}$ e $100 \mathrm{mg} \mathrm{dm}^{-3}$ de K, tendo como fontes $\mathrm{NaH}_{2} \mathrm{PO}_{4} \cdot \mathrm{H}_{2} \mathrm{O}, \mathrm{KCl}$ e $\mathrm{K}_{2} \mathrm{SO}_{4}$, conforme sugerido por Passos (1994), e uma solução de micronutrientes nas doses de $0,81 \mathrm{mg} \mathrm{dm}^{-3} \mathrm{de} \mathrm{B}\left(\mathrm{H}_{3} \mathrm{BO}_{3}\right)$, $1,33 \mathrm{mg} \mathrm{dm}^{-3} \mathrm{de} \mathrm{Cu}\left(\mathrm{CuSO}_{4} \cdot 5 \mathrm{H}_{2} \mathrm{O}\right), 0,15 \mathrm{mg} \mathrm{dm}^{-3}$ de $\left.\mathrm{Mo}\left[\left(\mathrm{NH}_{4}\right)_{6} \mathrm{Mo}_{7} \mathrm{O}_{24} \cdot 4 \mathrm{H}_{2} \mathrm{O}\right)\right], 3,66 \mathrm{mg} \mathrm{dm}^{-3}$ de $\mathrm{Mn}$

Tabela 1. Análise química do solo utilizado na produção das mudas de ipê-amarelo (Tabebuia serratifolia) antes da correção.

Table 1. Chemical analysis of the soil used in the production of seedlings of ipê-amarelo (Tabebuia serratifolia) before fertilizer application.

\begin{tabular}{|c|c|c|c|c|c|c|c|c|c|c|c|c|}
\hline \multirow{2}{*}{ Solo } & $\mathrm{pH}$ & \multirow{2}{*}{\multicolumn{2}{|c|}{$\frac{\mathrm{K}}{\mathrm{mg} \mathrm{dm}}$}} & $\mathrm{Ca}^{2+}$ & $\mathrm{Mg}^{2+}$ & $\mathrm{Al}^{3+}$ & $\mathrm{H}+\mathrm{Al}$ & SB & (T) & V & $\mathbf{m}$ & MO \\
\hline & $\mathrm{HO}$ & & & ----- & $-\overline{-----}$ & \multicolumn{3}{|c|}{$\mathrm{cmol} \mathrm{dm}^{-3}$} & ----- & & $\%$ & dag kg \\
\hline S SOlo & 4,79 & 0,7 & 6 & 0,11 & 0,01 & 0,92 & 3,9 & 0,14 & 4,04 & 3,5 & 86,8 & 1,66 \\
\hline
\end{tabular}

pH em água - Relação 1:2,5; P e K - Extrator Mehlich 1; $\mathrm{Ca}^{2+}, \mathrm{Mg}^{2+}$ e $\mathrm{Al}^{3+}$ - Extrator: $\mathrm{KCl} 1 \mathrm{~mol} / \mathrm{L} ; \mathrm{H}+\mathrm{Al}$ - Extrator acetato de cálcio $0,5 \mathrm{~mol} / \mathrm{L}$ - pH 7,0; SB - Soma de bases trocáveis; V - Índice de saturação por bases; $\mathrm{m}$ - Índice de saturação de alumínio; MO - C, Org $\times 1,724-$ Walkley-Black. 
$\left(\mathrm{MnCl}_{2} \cdot \mathrm{H}_{2} \mathrm{O}\right)$ e $4,0 \mathrm{mg} \mathrm{dm}-3$ de $\mathrm{Zn}\left(\mathrm{ZnSO}_{4} \cdot 7 \mathrm{H}_{2} \mathrm{O}\right)$ (Alvarez et al., 2006).

As sementes do ipê-amarelo (Tabebuia serratifolia) foram adquiridas no Laboratório de Análise de Sementes Florestais do Departamento de Engenharia Florestal da Universidade Federal de Viçosa. As sementes foram colocadas para germinar em sementeiras, com areia lavada como substrato. Após 15 dias da germinação, foram transplantadas, mantendo-se duas plântulas por vaso. Decorridos 30 dias, desbastou-se, deixando-se apenas uma planta por vaso. Os vasos foram mantidos em casa de vegetação e o teor de umidade do solo foi controlado diariamente, sendo mantido próximo a $60 \%$ da capacidade de campo.

A unidade experimental foi constituída por um vaso contendo $1,5 \mathrm{dm}^{3}$ de solo, com uma planta. Adotou-se um esquema fatorial $(3 \times 5)$, correspondendo a 3 fontes e 5 doses de nitrogênio, disposto no delineamento experimental casualizado em blocos, com quatro repetições, totalizando 60 vasos. As fontes de nitrogênio testadas foram nitrato de amônio $\left(\mathrm{NH}_{4} \mathrm{NO}_{3}\right)$, sulfato de amônio $\left[\left(\mathrm{NH}_{4}\right)_{2} \mathrm{SO}_{4}\right]$ e nitrato de cálcio $\left[\mathrm{Ca}\left(\mathrm{NO}_{3}\right)_{2}\right] \mathrm{em}$ cinco doses ( $0 \mathrm{mg}, 75 \mathrm{mg}, 150 \mathrm{mg}, 225 \mathrm{mg}$ e $300 \mathrm{mg} \mathrm{dm}^{-3}$ de $\mathrm{N}$ ), aplicadas como solução e divididas, cada dose, em quatro porções iguais aos 25, 50, 75 e 100 dias após a repicagem. Foram escolhidas essas fontes de nitrogênio porque elas apresentam em sua formulação o nitrogênio nítrico $\left(\mathrm{NO}_{3}^{-}\right)$e o amoniacal $\left(\mathrm{NH}_{4}^{+}\right)$, possibilitando a verificação da forma preferencial de absorção de $\mathrm{N}$ pela espécie teste.

Utilizando-se uma régua graduada em centímetros e um paquímetro digital graduado em milímetros, foram mensurados, 125 dias após a repicagem, respectivamente, a altura $(\mathrm{H})$ e o diâmetro do coleto (DC) das mudas. Logo após a tomada dos valores de altura e do diâmetro do coleto, a parte aérea e as raízes das plantas foram separadas e colocadas em estufa de circulação de ar a $65^{\circ} \mathrm{C}$ por três dias, para secagem.

Após o período de secagem na estufa, as partes das mudas foram pesadas em balança analítica (precisão), para se obterem os valores de matéria seca da parte aérea (PMSPA) e das raízes (PMSR). Com a soma do peso da matéria seca da parte aérea (PMSPA) e do das raízes (PMSR), obteve-se o peso da matéria seca total (PMST). A partir desses dados foi possível calcular a relação altura/diâmetro de coleto (RHDC), a relação altura/peso de matéria seca de parte aérea
(RHPMSPA), a relação peso de matéria seca da parte aérea/peso de matéria seca de raiz (RPMSPAR), e o Índice de Qualidade de Dickson (IQD), de acordo com a fórmula (Dickson et al., 1960 apud Gomes et al., 2002):

$\mathrm{IQD}=\frac{\mathrm{MST}(\mathrm{g})}{\mathrm{H}(\mathrm{cm}) / \mathrm{D}(\mathrm{mm})+\operatorname{MSPA}(\mathrm{g}) / \operatorname{MSR}(\mathrm{g})}$

Para as características quantitativas (H, DC, PMSPA, PMSR e PMST), foi determinada a dose crítica de N, que é a dosagem na qual se obtém $90 \%$ da produção máxima (Gonçalves et al., 2014). Nas relações obtidas (RHDC, RHPMSPA, RPMSPAR e IQD), calculou-se a dose de $\mathrm{N}$ na qual o índice máximo foi atingido.

Os dados foram analisados por meio de Análise de Variância (ANOVA) e pelo Teste de Tukey, utilizado para comparar o efeito principal das fontes de $\mathrm{N}$, e pela análise de regressão, para associar o efeito das doses. Nesse processo foi utilizado o programa Statistica 7.0. $\mathrm{Na}$ escolha das equações de regressão, considerou-se a significância dos coeficientes e o coeficiente de determinação ajustado $\left(\mathrm{R}^{2}\right)$. O nível de significância empregado em todas as análises foi de $5 \%$.

\section{RESULTADOS E DISCUSSÃO}

Aos 125 dias após a repicagem, observou-se efeito significativo da interação fontes de nitrogênio/doses de nitrogênio sobre a $\mathrm{H}$ (altura da parte aérea), DC (diâmetro do coleto), PMSPA (peso massa seca da parte aérea), PMSR (peso massa seca das raízes), PMST (peso massa seca total), RHPMSPA (relação altura da parte aérea/peso massa seca da parte aérea), RPMSPAR (relação peso massa seca da parte aérea/peso massa seca das raízes) e IQD (Índice de Qualidade de Dickson), mas não para a RHDC (relação altura da parte aérea/diâmetro do coleto) (Tabela 2). Ao desdobrar a interação, a fonte nitrato de cálcio não apresentou efeito significativo sobre as características e as relações avaliadas. Para as doses de nitrogênio (N) de cada fonte utilizada, foram ajustadas equações de regressão e, a partir delas, calculadas as doses críticas e máximas de cada característica e índice avaliados.

Para $\mathrm{H}$ e DC, as fontes de $\mathrm{N}$ aplicadas não diferiram entre si pelo teste de Tukey*. Para as demais características e relações estudadas, as fontes de $\mathrm{N}$ na forma de nitrato de amônio e sulfato de amônio não diferiram entre si pelo teste de Tukey*. Apenas o nitrato 
de cálcio exerceu efeitos diferenciados em relação às outras fontes de $\mathrm{N}$, apresentando as menores médias.

As médias dos parâmetros de crescimento e biomassa estão apresentadas na Tabela 3. As maiores médias das características avaliadas obtidas pelas mudas que receberam nitrato de amônio e sulfato de amônio devem-se à presença da forma amoniacal do $\mathrm{N}$, evidenciando a preferência dessa espécie de absorver nitrogênio nessa forma em detrimento da forma nítrica, presente no nitrato de cálcio. Comportamento semelhante foi observado por Marques et al. (2006a) em mudas de jacarandá-da-Bahia, que receberam as mesmas fontes de $\mathrm{N}$ do presente estudo e também apresentaram preferência pela absorção de $\mathrm{N}$ na forma amoniacal (sulfato de amônio) à forma nítrica, evidenciado pelas maiores médias das características avaliadas nas mudas que receberam sulfato de amônio.

Para a altura da parte aérea (Figura 1), o nitrato de amônio e o sulfato de amônio proporcionaram efeito quadrático das doses de $\mathrm{N}$ aplicadas, possibilitando a determinação da dose crítica de $136 \mathrm{mg} \mathrm{dm}^{-3} \mathrm{e}$ $96 \mathrm{mg} \mathrm{dm}^{-3}$ de $\mathrm{N}$, respectivamente. Efeito linear positivo da aplicação de doses de $\mathrm{N}$ sobre a altura da parte aérea também foi verificado em mudas de sabiá (Mimosa caesalpiniaefolia) (Marques et al., 2006b; Gonçalves et al., 2013) e em mudas de Schizolobium parahyba (Vieira et al., 2007) sendo que a última espécie apresentou a maior altura e diâmetro de colo com a aplicação de $100 \mathrm{mg} \mathrm{dm}^{-3} \mathrm{de} \mathrm{N}$.

A presença do enxofre na fonte de $\mathrm{N}$ sulfato de amônio não influencia o crescimento das mudas de ipê-amarelo, pois já foi verificado que em espécies nativas como jacarandá-da-Bahia (Gonçalves et al., 2014) e canafístula (Cruz et al., 2012) esse nutriente é pouco requerido na fase de formação das mudas.

Com base da mensuração do diâmetro do coleto (Figura 2), as doses críticas encontradas para nitrato de amônio e sulfato de amônio foram $254 \mathrm{mg} \mathrm{dm}^{-3} \mathrm{e}$

Tabela 2. Resumo da análise de variância das características quantitativas e relações estudadas na produção de mudas de ipê-amarelo (Tabebuia serratifolia), avaliadas 125 dias após a repicagem.

Table 2. Summary of analysis of variance for the studied morphological characteristics and relationships of ipê-amarelo (Tabebuia serratifolia), evaluated at 125 days after transplanting.

\begin{tabular}{|c|c|c|c|c|c|c|c|c|c|c|}
\hline \multirow{2}{*}{ FV } & \multirow{2}{*}{ GL } & \multicolumn{9}{|c|}{ Quadrado médio } \\
\hline & & $\mathbf{H}$ & DC & PMSPA & PMSR & PMST & RHDC & RHPMSPA & RPMSPAR & IQD \\
\hline Bloco & 3 & $12,28^{\mathrm{ns}}$ & $0,311^{\mathrm{ns}}$ & $0,202^{\text {ns }}$ & $0,196^{\mathrm{ns}}$ & $0,821^{\mathrm{ns}}$ & $0,985^{\mathrm{ns}}$ & $135,1^{\mathrm{ns}}$ & $0,0138^{\text {ns }}$ & $0,053^{\mathrm{ns}}$ \\
\hline Fonte (F) & 2 & $33,198^{*}$ & $0,1624^{*}$ & $1,3882^{*}$ & $1,8841^{*}$ & $3,1373^{*}$ & $1,5134^{\mathrm{ns}}$ & $623,6^{*}$ & $0,623^{*}$ & $1,3972^{*}$ \\
\hline Doses (D) & 4 & $94,886^{*}$ & $3,5912^{*}$ & $1,9835^{\star}$ & $2,384^{*}$ & $5,2576^{*}$ & $0,242^{\mathrm{ns}}$ & $1795,7^{\star}$ & $0,5417^{\star}$ & $1,8090^{*}$ \\
\hline$F \times D$ & 8 & $31,754^{*}$ & $1,2892^{*}$ & $0,8399^{*}$ & $0,9399^{*}$ & $2,2677^{\star}$ & $0,819^{\text {ns }}$ & $712^{*}$ & $0,4931^{*}$ & $0,756^{*}$ \\
\hline Resíduo & 42 & 8,873 & 0,3164 & 0,1599 & 0,2118 & 0,5545 & 1,534 & 253,7 & 0,1095 & 0,0975 \\
\hline $\mathrm{CV} \%$ & & 22,97 & 22,47 & 52,43 & 66,72 & 55,66 & 23,07 & 55,96 & 25,45 & 19,59 \\
\hline
\end{tabular}

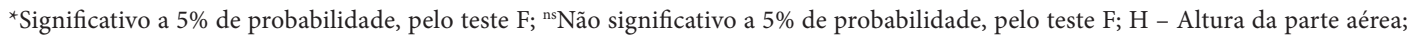
DC - Diâmetro do coleto; PMSPA - Peso de matéria seca da parte aérea; PMSR - Peso da matéria seca das raízes; PMST - Peso da matéria seca total; RHDC - Relação altura da parte aérea/diâmetro do coleto; RHPMSPA - Relação altura da parte aérea/peso da matéria seca da parte aérea; RPMSPAR - Relação peso da matéria seca da parte aérea/peso da matéria seca das raízes; IQD - Índice de Qualidade de Dickson.

Tabela 3. Médias observadas para o crescimento em altura $(\mathrm{H} ; \mathrm{cm})$, diâmetro do coleto (DC; mm), massa seca da parte aérea (PMSPA; g), massa seca das raízes (PMSR; g) e massa seca total (PMST; g) de mudas de ipê-amarelo (Tabebuia serratifolia) em resposta às doses de nitrogênio $\left(\mathrm{mg} / \mathrm{dm}^{3}\right)$, para as três fontes de $\mathrm{N}$ estudadas (nitrato de amônio, sulfato de amônio e nitrato de cálcio), 125 dias após a repicagem.

Table 3. Observed averages for shoot height (H; cm), stem diameter (DC; mm), shoot dry mass (PMSPA; g), roots dry mass (PMSR; g) and total dry mass (PMST; g) seedlings of ipê-amarelo (Tabebuia serratifolia), in response to nitrogen levels $\left(\mathrm{mg} / \mathrm{dm}^{3}\right.$ ), for the three $\mathrm{N}$ sources studied (ammonium nitrate, ammonium sulfate and calcium nitrate) at 125 days after transplanting.

\begin{tabular}{lccccc}
\multicolumn{1}{c}{ Fontes de nitrogênio } & $\mathbf{H}(\mathbf{c m})$ & $\mathbf{D C}(\mathbf{m m})$ & PMSPA $(\mathbf{g})$ & PMSR (g) & PMST (g) \\
\hline Nitrato de amônio & 13,22 & 2,55 & 0,87 & 0,74 & 1,61 \\
Sulfato de amônio & 16,04 & 2,56 & 1,25 & 1,02 & 2,27 \\
Nitrato de cálcio & 11,72 & 2,26 & 0,46 & 0,42 & 0,88 \\
\hline
\end{tabular}




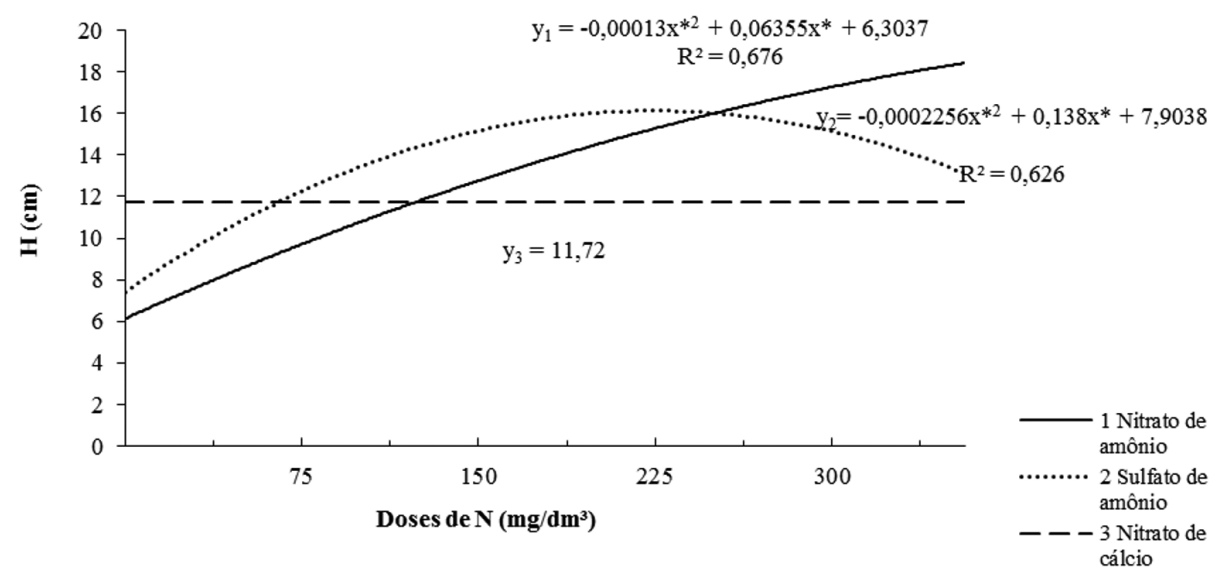

Figura 1. Altura da parte aérea $(\mathrm{H})$ de mudas de ipê-amarelo (Tabebuia serratifolia) em resposta a doses de nitrogênio $\left(\mathrm{mg} / \mathrm{dm}^{3}\right)$, para as três fontes de $\mathrm{N}$ estudadas (nitrato de amônio, sulfato de amônio e nitrato de cálcio), 125 dias após a repicagem.

Figure 1. Shoot height (H) of ipê-amarelo (Tabebuia serratifolia) seedlings, in response to nitrogen levels $\left(\mathrm{mg} / \mathrm{dm}^{3}\right)$, for the three $\mathrm{N}$ sources studied (ammonium nitrate, ammonium sulfate and calcium nitrate) at 125 days after transplanting.

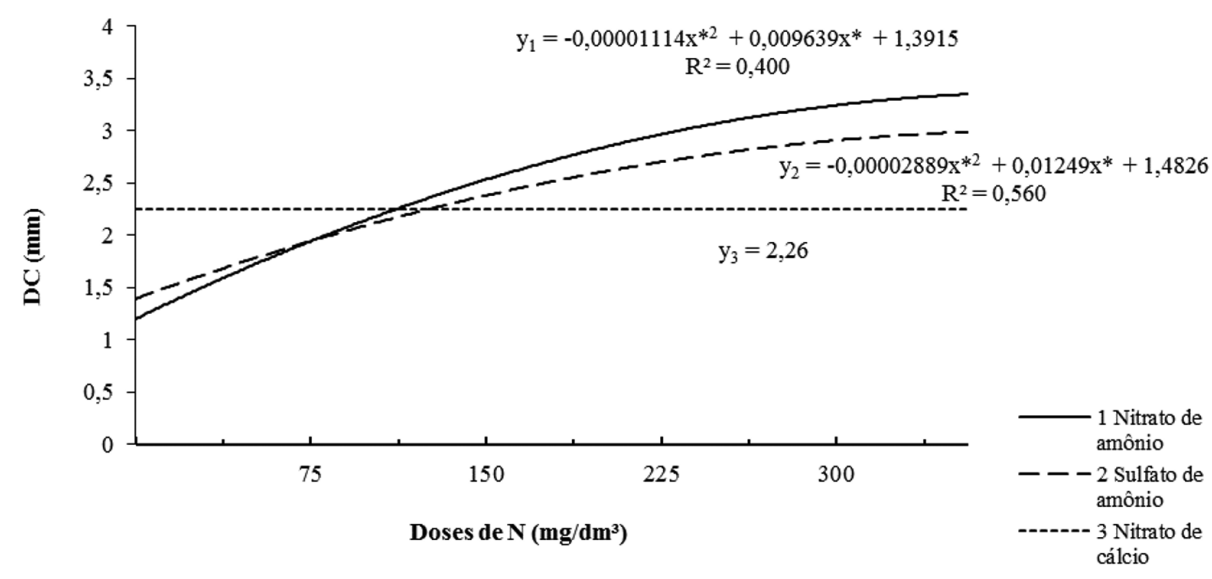

Figura 2. Diâmetro do coleto (DC) de mudas de ipê-amarelo (Tabebuia serratifolia) em resposta a doses de nitrogênio $\left(\mathrm{mg} / \mathrm{dm}^{3}\right)$, para as três fontes de $\mathrm{N}$ estudadas (nitrato de amônio, sulfato de amônio e nitrato de cálcio), 125 dias após a repicagem.

Figure 2. Stem diameter (DC) of ipê-amarelo (Tabebuia serratifolia) seedlings, in response to nitrogen levels $\left(\mathrm{mg} / \mathrm{dm}^{3}\right)$, for the three $\mathrm{N}$ sources studied (ammonium nitrate, ammonium sulfate and calcium nitrate) at 125 days after transplanting.

$118 \mathrm{mg} \mathrm{dm}^{-3} \mathrm{de} \mathrm{N}$, respectivamente. Efeito quadrático positivo para a aplicação de $\mathrm{N}$ foi observado sobre diâmetro do coleto, utilizando-se as fontes nitrato de amônio e sulfato de amônio, em mudas de jacarandá-da-Bahia (Dalbergia nigra) (Marques et al., 2006b). Já em mudas de Peltophorum dubium, a aplicação de $\mathrm{N}$ proporcionou efeito linear negativo, indicando que, de modo geral, maior crescimento e melhor qualidade das mudas de canafístula serão obtidos com aplicação de doses inferiores a $50 \mathrm{mg} \mathrm{dm}-3$ de N (Cruz et al., 2012).

As doses críticas encontradas para nitrato de amônio e sulfato de amônio no PMSPA (Figura 3) foram $136 \mathrm{mg} \mathrm{dm}^{-3}$ e $113 \mathrm{mg} \mathrm{dm}^{-3}$ de $\mathrm{N}$, respectivamente. Em mudas de sansão-do-campo (Mimosa caesalpiniaefolia), em resposta à aplicação de nitrogênio foi observado comportamento semelhante ao da espécie deste 
estudo, na qual o efeito quadrático da aplicação de $\mathrm{N}$ observado no Latossolo Vermelho-Amarelo sobre a altura e PMSPA sugere aumento dos valores dessas características à medida que se aumentam as doses (Gonçalves et al., 2010).

Em relação às raízes (Figura 4), o nitrato de amônio apresentou efeito quadrático em resposta à aplicação das doses de nitrogênio, sendo a dose crítica atingida em 99, $6 \mathrm{mg} \mathrm{dm}^{-3}$ de N. Sulfato de amônio e nitrato de cálcio não proporcionaram efeito significativo para as doses de N sobre PMSR. Resultados diferentes foram encontrados por Gonçalves et al. (2008) em mudas de angico-vermelho (Anadenanthera macrocarpa), em que a aplicação de $\mathrm{N}$ não apresentou efeito significativo sobre PMSR.

Para PMST (Figura 5), nitrato de amônio e sulfato de amônio apresentaram doses críticas de $117 \mathrm{mg} \mathrm{dm}^{-3}$ e $111 \mathrm{mg} \mathrm{dm}^{-3}$ de $\mathrm{N}$, respectivamente.

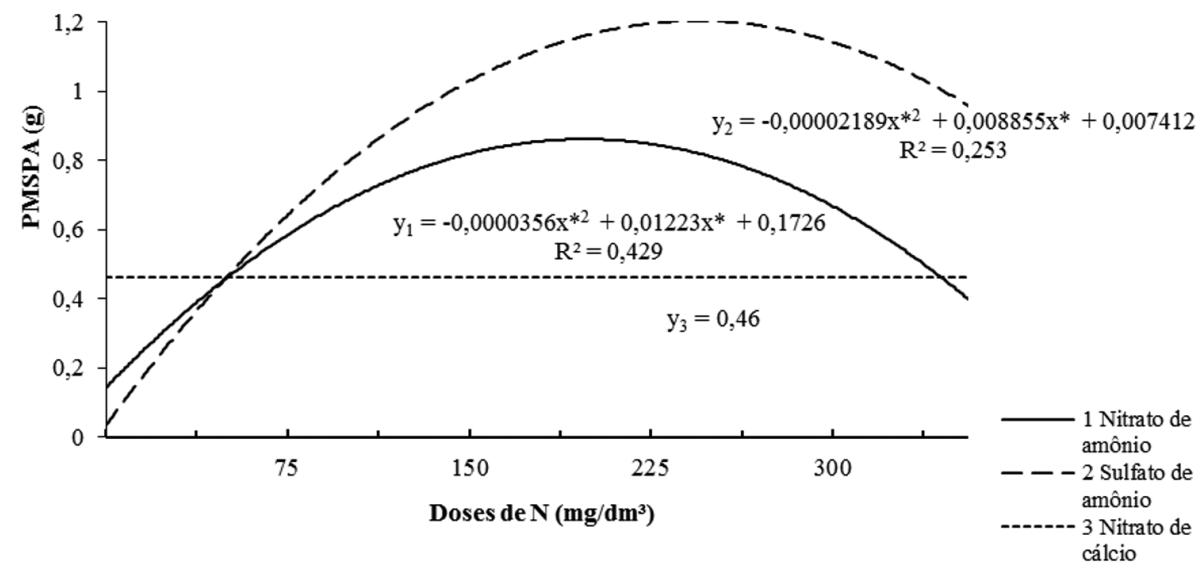

Figura 3. Massa seca da parte aérea (PMSPA) de mudas de ipê-amarelo (Tabebuia serratifolia) em resposta a doses de nitrogênio $\left(\mathrm{mg} / \mathrm{dm}^{3}\right)$, para as três fontes de $\mathrm{N}$ estudadas (nitrato de amônio, sulfato de amônio e nitrato de cálcio), 125 dias após a repicagem.

Figure 3. Shoot dry mass (PMSPA) of ipê-amarelo (Tabebuia serratifolia) seedlings, in response to nitrogen levels $\left(\mathrm{mg} / \mathrm{dm}^{3}\right)$, for the three $\mathrm{N}$ sources studied (ammonium nitrate, ammonium sulfate and calcium nitrate) at 125 days after transplanting.

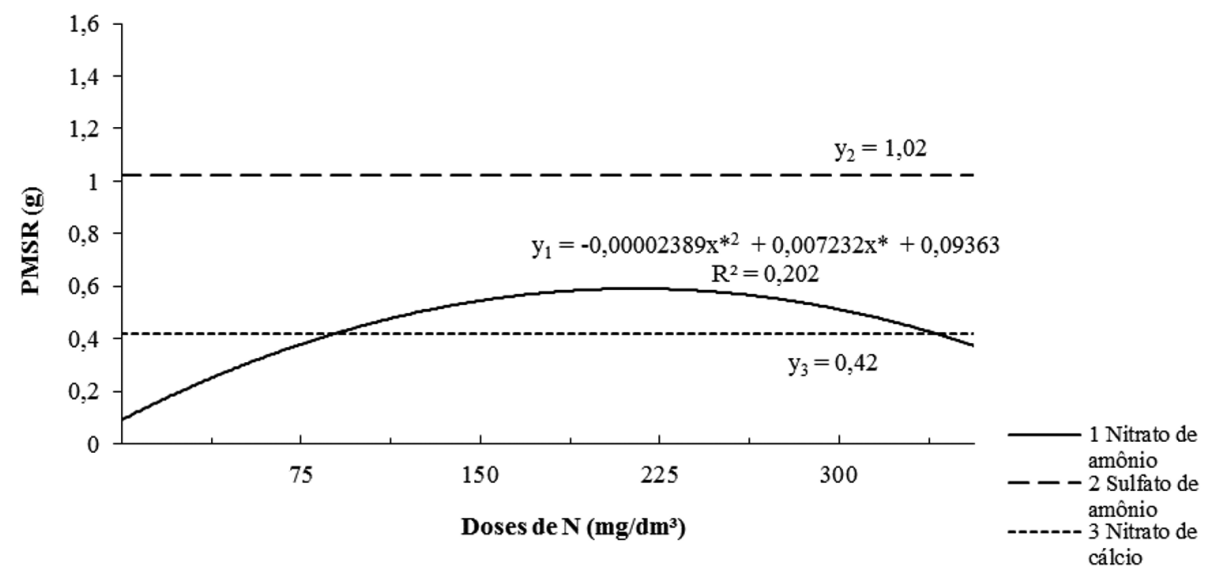

Figura 4. Massa seca das raízes (PMSR) de mudas de ipê-amarelo (Tabebuia serratifolia) em resposta a doses de nitrogênio $\left(\mathrm{mg} / \mathrm{dm}^{3}\right)$, para as três fontes de $\mathrm{N}$ estudadas (nitrato de amônio, sulfato de amônio e nitrato de cálcio), 125 dias após a repicagem.

Figure 4. Roots dry mass (PMSR) of ipê-amarelo (Tabebuia serratifolia) seedlings, in response to nitrogen levels $\left(\mathrm{mg} / \mathrm{dm}^{3}\right)$, for the three $\mathrm{N}$ sources studied (ammonium nitrate, ammonium sulfate and calcium nitrate) at 125 days after transplanting. 
Cruz et al. (2006), estudando o efeito da adubação nitrogenada sobre o crescimento e qualidade das mudas de sete-cascas (Samanea inopinata), verificaram resultados semelhantes em relação ao PMST, em que o sulfato de amônio também foi a melhor fonte de $\mathrm{N}$, apresentando maiores médias em relação ao nitrato de amônio e ao nitrato de cálcio. Em Cedrela fissilis, a aplicação de $\mathrm{N}$ também proporcionou efeito linear positivo sobre o PMST (Freiberger et al., 2013).

Nitrato de amônio e sulfato de amônio proporcionaram resposta quadrática na função doses de N/RHPMSPA
(Tabela 4). Mudas de Eucalyptus grandis, aos 90 dias após a semeadura, em resposta à fertilização com N-P-K, apresentaram efeitos significativos nas RHPMSPA e no IQD (Gomes et al., 2003).

A RPMSPAR expressa o grau de equilíbrio de alocação de carbono nas plantas e seu índice ideal é 2 (Marques et al., 2006b). Para nitrato de amônio e sulfato de amônio, os valores máximos dos índices RPMSPAR foram de 2,05 na dose de $540 \mathrm{mg} \mathrm{dm}^{-3} \mathrm{e}$ 1,7 na dose de $196 \mathrm{mg} \mathrm{dm}^{-3}$ de $\mathrm{N}$, respectivamente, calculados através das equações de regressão geradas.

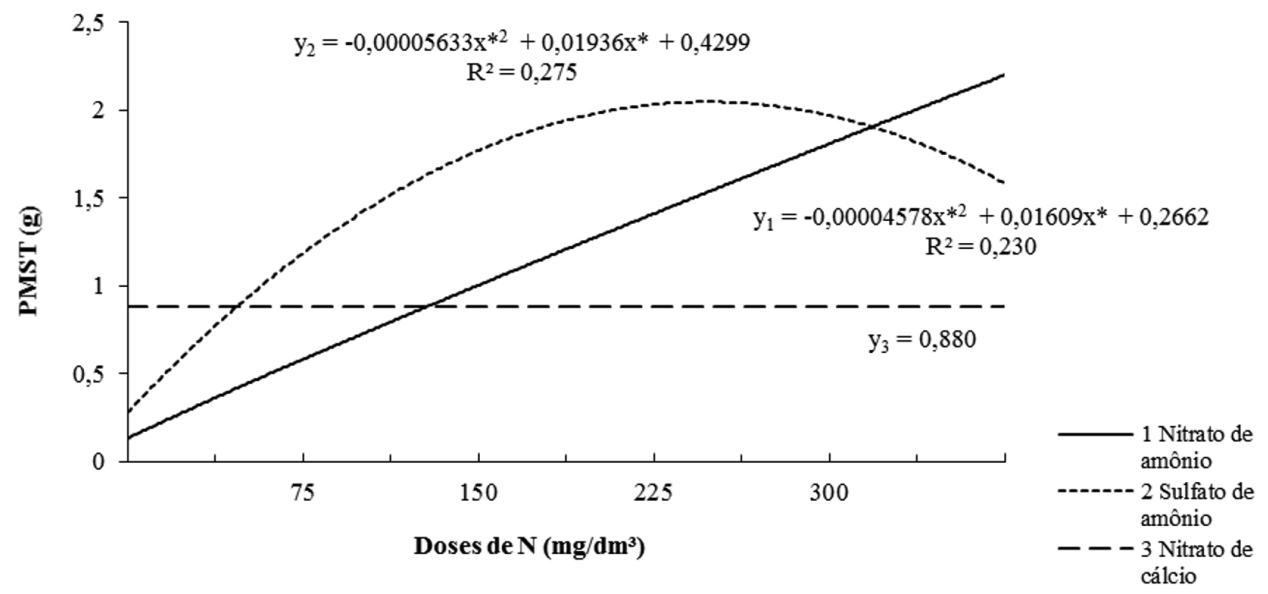

Figura 5. Massa seca total (PMST) de mudas de ipê-amarelo (Tabebuia serratifolia) em resposta a doses de nitrogênio $\left(\mathrm{mg} / \mathrm{dm}^{3}\right)$, para as três fontes de $\mathrm{N}$ estudadas (nitrato de amônio, sulfato de amônio e nitrato de cálcio), 125 dias após a repicagem. ${ }^{\star}$ Significativo a $5 \%$ de probabilidade.

Figure 5. Total dry mass (PMST) of ipê-amarelo (Tabebuia serratifolia) seedlings, in response to nitrogen levels $\left(\mathrm{mg} / \mathrm{dm}^{3}\right)$, for the three $\mathrm{N}$ sources studied (ammonium nitrate, ammonium sulfate and calcium nitrate) at 125 days after transplanting. ${ }^{*}$ Significant at $5 \%$ probability.

Tabela 4. Equações de regressão geradas do efeito de fontes (nitrato de amônio, sulfato de amônio e nitrato de cálcio) e doses de N (0 mg, $75 \mathrm{mg}, 150 \mathrm{mg}, 225 \mathrm{mg}$ e $300 \mathrm{mg} \mathrm{dm}^{-3}$ ) sobre as relações estudadas, em mudas de ipê-amarelo (Tabebuia serratifolia), avaliadas 125 dias após a repicagem.

Table 4. Equations generated estimations from the effect of sources (ammonium nitrate, ammonium sulphate and calcium nitrate) and $\mathrm{N}$ levels $\left(0,75,150,225\right.$ e $\left.300 \mathrm{mg} \mathrm{dm}^{-3}\right)$ on relations studied, in ipê-amarelo (Tabebuia serratifolia) seedlings, evaluated at 125 days after transplanting.

\begin{tabular}{|c|c|c|c|}
\hline Fontes & Características & ${ }^{(1-9)}$ Equações de regressão & $\mathbf{R}^{2}$ \\
\hline \multirow{3}{*}{ Nitrato de amônio } & RHPMSPA & ${ }^{1} \hat{Y}=57,6017-0,2148 x^{\star}+0,0001777 x^{\star 2}$ & 0,414 \\
\hline & RPMSPAR & ${ }^{2} \hat{Y}=0,9097+0,004288 x^{\star}-0,00000397 x^{\star 2}$ & 0,343 \\
\hline & IQD & ${ }^{3} \hat{Y}=0,9860+0,005002 x^{\star}-0,0000035 x^{\star 2}$ & 0,568 \\
\hline \multirow{3}{*}{ Sulfato de amônio } & RHPMSPA & ${ }^{4} \hat{Y}=47,4962-0,3157 x^{*}+0,0006485 x^{\star 2}$ & 0,444 \\
\hline & RPMSPAR & ${ }^{5} \hat{Y}=0,8136+0,008954 x^{\star}-0,000002288 x^{\star 2}$ & 0,358 \\
\hline & IQD & ${ }^{6} \hat{Y}=0,9356+0,009061 x^{*}-0,00001562 x^{* 2}$ & 0,598 \\
\hline \multirow{3}{*}{ Nitrato de cálcio } & RHPMSPA & ${ }^{7} \hat{Y}=\ddot{Y}=31,98$ & - \\
\hline & RPMSPAR & ${ }^{8} \hat{Y}=\ddot{Y}=1,09$ & - \\
\hline & IQD & ${ }^{9} \hat{Y}=\ddot{Y}=1,29$ & - \\
\hline
\end{tabular}

*Significativo a 5\% de probabilidade. $\mathrm{R}^{2}$ - Coeficiente de determinação ajustado; RHDC - Relação altura/diâmetro do coleto; RHPMSPA - Relação altura/peso da matéria seca de parte aérea; RPMSPAR - Relação peso da matéria seca de parte aérea/peso da matéria seca das raízes; IQD - Índice de Qualidade de Dickson. 
Para se obter índice máximo na RPMSPAR com a utilização da fonte nitrato de cálcio é necessário utilizar uma dose superior à dose máxima de $\mathrm{N}$ aqui estudada.

Marques et al. (2009), estudando o efeito de diferentes tipos de solos, fontes e doses de nitrogênio em mudas de jacaré (Piptadenia gonoacantha), concluíram que a RPMSPAR não foi influenciada pelas fontes nitrogenadas, observando-se efeito significativo apenas na interação solos-doses. Tucci et al. (2009), estudando efeitos da adubação nitrogenada na produção de mudas de mogno (Swietenia macrophylla) também não observaram efeito das doses crescentes de N sobre RPMSPAR.

No IQD, quanto maior for o valor do índice, melhor será a qualidade da muda produzida (Gomes et al., 2002). Para o ipê-amarelo, os maiores valores para esse índice foram encontrados com a aplicação de nitrato de amônio, com índice máximo obtido na dose além de $300 \mathrm{mg} \mathrm{dm}^{-3}$ de $\mathrm{N}$, e de sulfato de amônio, com 2,3 de índice máximo, na dose de $290 \mathrm{mg} \mathrm{dm}^{-3}$ de N. Em mudas de fedegoso (Senna macranthera), o IQD apresentou respostas lineares crescentes ao tratamento com nitrogênio, com maiores índices em doses superiores a $170 \mathrm{mg} \mathrm{dm}^{-3}$ de N (Cruz et al., 2010).

\section{CONCLUSÕES}

As mudas de ipê-amarelo (Tabebuia serratifolia) responderam significativamente à adição do nitrogênio mineral, tais como sulfato de amônio e nitrato de amônio, apresentando ganhos em crescimento, qualidade e evidenciando a preferência dessa espécie de absorver $\mathrm{N}$ na forma amoniacal.

As melhores médias para todas as características quantitativas são obtidas com aplicação de $\mathrm{N}$ variando entre $90 \mathrm{mg}$ e $110 \mathrm{mg} \mathrm{dm}^{-3}$ de $\mathrm{N}$.

Recomenda-se, na produção de mudas de ipê-amarelo (Tabebuia serratifolia), a dose de $100 \mathrm{mg} \mathrm{dm}^{-3}$ de N, aplicada parceladamente aos 25, 50, 75 e 100 dias e tendo como fonte de $\mathrm{N}$ o sulfato de amônio.

\section{STATUS DA SUBMISSÃO}

Recebido: 16 mar., 2015

Aceito: 22 mar., 2016

\section{AUTOR(ES) PARA CORRESPONDÊNCIA}

\section{Lívia Mara Lima Goulart}

Departamento de Solos e Recursos Ambientais, Universidade Estadual Paulista - UNESP, Rua José Barbosa de Barros, 1780, Fazenda Lageado, CEP 18610-370, Botucatu, SP, Brasil e-mail: liviamlgoulart@yahoo.com.br

\section{REFERÊNCIAS}

Alvarez V, Dias LE, Leite PB, Souza RB, Ribeiro ESR Jr. Poda de raízes e adubação para crescimento do cafeeiro cultivado em colunas de solo. Revista Brasileira de Ciencia do Solo 2006; 30(1): 111-119. http://dx.doi.org/10.1590/ S0100-06832006000100012.

Cruz CAF, Paiva HN, Cunha ACMCM, Neves JCL. Resposta de mudas de Senna macranthera cultivadas em Argissolo vermelho-amarelo a macronutrientes. Ciência Florestal 2010; 21(1): 63-76. http://dx.doi.org/10.5902/19805098.

Cruz CAF, Paiva HN, Cunha ACMCM, Neves JCL. Produção de mudas de canafístula cultivadas em Latossolo vermelho amarelo álico em resposta a macronutrientes. Cerne 2012; 18(1): 87-98. http://dx.doi.org/10.1590/ S0104-77602012000100011.

Cruz CAF, Paiva HN, Guerrero CA. Efeito da adubação nitrogenada na produção de mudas de sete-cascas (Samanea inopinata (Harms) Ducke). Revista Árvore 2006; 30(4): 537-546. http://dx.doi.org/10.1590/S010067622006000400006

Cruz GL. Livro verde das plantas medicinais e industriais do Brasil. Belo Horizonte: Velloso; 1965. vol. 2.

Ferreira L, Chalub D \& Muxfeldt R. Ipê-amarelo (Tabebuia serratifolia (Vahl) Nichols.). Informativo Técnico Rede de Sementes da Amazônia 2004; 5.

Freiberger MB, Guerrini IA, Galetti G, Fernandes DM, Corrêa JC. Crescimento inicial e nutrição de cedro (Cedrela fissilis Vell.) em função de doses de nitrogênio. Revista Árvore 2013; 37(3): 385-392. http://dx.doi.org/10.1590/ S0100-67622013000300001.

Gomes JM, Couto L, Leite HG, Xavier A, Garcia SLR. Parâmetros morfológicos na avaliação da qualidade de mudas de Eucalyptus grandis. Revista Árvore 2002; 26(6): 655-664. http://dx.doi.org/10.1590/S0100-67622002000600002.

Gomes JM, Couto L, Leite HG, Xavier A, Garcia SLR. Crescimento de mudas de Eucalyptus grandis em diferentes tamanhos de tubetes e fertilização N-P-K. Revista Árvore 2003; 27(2): 115-129. http://dx.doi.org/10.1590/S010067622003000200001 .

Gonçalves EO, Paiva HN, Neves JCL, Gomes JM. Crescimento de mudas de angico-vermelho (Anadenanthera macrocarpa (Benth.) Brenan) sob diferentes doses de 
macronutrientes. Revista Árvore 2008; 32(6): 1029-1040. http://dx.doi.org/10.1590/S0100-67622008000600008.

Gonçalves EO, Paiva HN, Neves JCL, Gomes JM. Crescimento de mudas de sansão-do-campo (Mimosa caesalpiniaefolia Benth.) sob diferentes doses de macronutrientes. Scientia Forestalis 2010; 38(88): 599-609. http://dx.doi.org/10.1590/ S0100-67622014000200005.

Gonçalves EO, Paiva HN, Neves JCL, Gomes JM. Nutrição de mudas de Mimosa caesalpiniaefolia Benth. sob diferentes doses de N,P, K, Ca e Mg. Ciência Florestal 2013; 23(2): 273-286. http://dx.doi.org/10.5902/198050989274.

Gonçalves EO, Paiva HN, Neves JCL, Klippel VH, Caldeira MVW. Crescimento de Dalbergia nigra (Vell.) Allemão ex Benth sob diferentes doses de cálcio, magnésio e enxofre. Revista Árvore 2014; 38(2): 251-260. http://dx.doi. org/10.1590/S0100-67622014000200005.

Gonçalves JLM, Stape JL, Benedetti V, Fessel VAG, Gava JL. Produção de mudas de espécies nativas: substrato, nutrição, sombreamento e fertilização. In: Gonçalves JLM, Benedetti V. Nutrição e fertilização florestal. Piracicaba: IPEF; 2000. p. 309-350.

Lorenzi H. Árvores brasileiras: manual de identificação e cultivo de plantas arbóreas nativas do Brasil. Nova Odessa: Plantarum; 1992. $352 \mathrm{p}$.

Marques LS, Paiva HN, Neves JCL, Gomes JM, Souza PH. Crescimento de mudas de jacaré (Piptadenia gonoacantha J.F. Macbr.) em diferentes tipos de solos e fontes e doses de nitrogênio. Revista Árvore 2009; 33(1): 81-92. http:// dx.doi.org/10.1590/S0100-67622009000100009.

Marques VB, Paiva HN, Gomes JM, Neves JCL, Bernardino DCS. Efeito de fontes e doses de nitrogênio sobre o crescimento inicial e qualidade de mudas de jacarandá-dabahia (Dalbergia nigra (Vell.) Fr. All. ex Benth.). Revista Árvore 2006a; 30(5): 725-735. http://dx.doi.org/10.1590/ S0100-67622006000500006.

Marques VB, Paiva HN, Gomes JM, Neves JCL. Efeitos de fontes e doses de nitrogênio no crescimento de mudas de sabiá (Mimosa caesalpiniaefolia Benth.). Scientia Forestalis 2006b; 71: 77-85.

Oliveira LM, Carvalho MLM, Silva TTA, Borges DI. Temperatura e regime de luz na germinação de sementes de Tabebuia impetiginosa (Martius ex A. P. de Candolle) Standley e T. serratifolia Vahl Nich. - Bignoniaceae. Ciência e Agrotecnologia 2005; 29(3): 642-648. http:// dx.doi.org/10.1590/S1413-70542005000300020.

Passos MAA. Efeito da calagem e de fósforo no crescimento inicial da algaroba (Prosopis juliflora (SW) DC) [tese]. Viçosa: Universidade Federal de Viçosa; 1994.

Souza CAM, Oliveira RB, Martins S Fo, Lima JSS. Crescimento em campo de espécies florestais em diferentes condições de adubações. Ciência Florestal. Santa Maria 2006; 16(3): 243-249. http://dx.doi.org/10.5902/19805098.

Tucci CAF, Lima HN, Lessa JF. Adubação nitrogenada na produção de mudas de mogno (Swietenia macrophylla King). Acta Amazonica 2009; 39(2): 289-294. http://dx.doi. org/10.1590/S0044-59672009000200007.

Vieira AH, Locatelli M, França JM, Carvalho JOM. Crescimento de mudas de Schizolobium parahyba var. amazonicum (Huber ex Ducke) Barneby sob diferentes niveis de nitrogênio, fósforo e potássio. Porto Velho: Embrapa Rondônia; 2007. Série Boletim de Pesquisa e Desenvolvimento. 